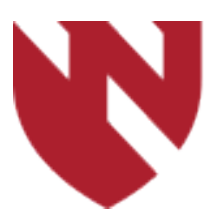

December 2019

\title{
Identifying Current Opioid Prescription Practice and Implementing Guidelines after Hand and Upper Extremity Surgery - A Project in Quality Improvement
}

\author{
Ryan E. Miller \\ University of Nebraska Medical Center \\ David Brown \\ University of Nebraska Medical Center \\ Mark Amirtharaj \\ University of Nebraska Medical Center \\ Brandon Bennett \\ University of Nebraska Medical Center \\ Todd Gillbert \\ University of Nebraska Medical Center
}

Tell us how you used this information in this short survey.

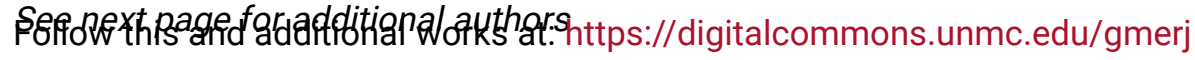

Part of the Higher Education Commons, and the Medicine and Health Sciences Commons

\section{Recommended Citation}

Miller, R. E., Brown, D., Amirtharaj, M., Bennett, B., Gillbert, T., Hansen, C., Larson, E., Rinehart, K., Firestone, D., Morgan, J., Streubel, P., , Teusink, M. Identifying Current Opioid Prescription Practice and Implementing Guidelines after Hand and Upper Extremity Surgery - A Project in Quality Improvement. Graduate Medical Education Research Journal. 2019 Dec 13; 1(1).

https://digitalcommons.unmc.edu/gmerj/vol1/iss1/37

This Conference Proceeding is brought to you for free and open access by DigitalCommons@UNMC. It has been accepted for inclusion in Graduate Medical Education Research Journal by an authorized editor of DigitalCommons@UNMC.For more information, please contact digitalcommons@unmc.edu. 


\section{Identifying Current Opioid Prescription Practice and Implementing Guidelines after Hand and Upper Extremity Surgery - A Project in Quality Improvement}

\section{Creative Commons License}

\section{(c) (i) $\Theta \Theta$}

This work is licensed under a Creative Commons Attribution-Noncommercial-No Derivative Works 4.0 License.

Authors

Ryan E. Miller, David Brown, Mark Amirtharaj, Brandon Bennett, Todd Gillbert, CJ Hansen, Evan Larson, Kent Rinehart, Daniel Firestone, Joseph Morgan, Philipp Streubel, and Matthew Teusink 


\section{Does Prophylactic Administration of TXA Reduce Mean Operative Time and Postoperative Blood Loss in Posterior Approach Lumbar Spinal Fusion Surgery Performed for Degenerative Spinal Disease?}

Evan Larson, Emmett Gannon, Tyler Evans, Jake Long, Elizabeth Lyden, Chris Cornett

Mentor: Chris Cornett

Program: Orthopaedic Surgery

Objective: To investigate association between prophylactic TXA administration prior to one and two-level posterior lumbar interbody fusion operations and perioperative blood loss, mean operative time, postoperative transfusion, and postoperative venous thromboembolic events.

Summary of Background Data: Tranexamic acid (TXA) is a systemic anti-fibrinolytic that competitively inhibits lysine binding sites on plasminogen, reversibly blocking its binding to fibrin and impeding fibrinolysis and clot degradation. TXA's role in routine spinal surgery remains poorly described. Most spinal literature on perioperative TXA administration has considered operations performed for major adult and pediatric spinal deformity.

Methods: Two groups, a study group composed of 75 patients who underwent one and two-level posterior lumbar interbody fusion operations for degenerative indications who received TXA prior to the start of the procedure, and a control group composed of 75 patients who underwent similar surgeries for the same indications and did not receive TXA preoperatively, were retrospectively enrolled. Demographic, laboratory, and surgical data were collected and analyzed.
Results: Statistically significant reductions were noted in the TXA group in postoperative day 1 drain output ( $\mathrm{p}<0.0041)$, total postoperative drain output $(\mathrm{p}=0.027)$, and mean surgical time $(\mathrm{p}<0.0001)$.

Conclusions: Based on the results of the current study, we feel TXA's routine use should be considered in one and two- level posterior lumbar fusion operations performed for degenerative indications, as it is safe to administer routinely and associated with reduced postoperative drain output and mean surgical time.

https://doi.org/10.32873/unmc.dc.gmerj.1.1.036

\section{Identifying Current Opioid Prescription Practice and Implementing Guidelines after Hand and Upper Extremity Surgery - A Project in Quality Improvement}

Ryan E Miller, David Brown, Mark Amirtharaj, Brandon Bennett, Todd Gilbert, CJ Hansen, Evan Larson, Kent Rinehart, Daniel Firestone, Joseph Morgan, Philipp Streubel, Matthew Teusink

\section{Mentor: Daniel Firestone}

Program: Orthopaedic Surgery

Background \& Objectives: Opioid pain medications prescriptions following upper extremity surgery is part of the standard of care. However, there is a growing concern about opioid abuse, overdose deaths, and diversion of opioids in the community. New evidence suggesting opioid overprescription has prompted a closer look at current practice. Objectives of this quality improvement project are 1) to define prescribing habits and patient utilization of opioids following upper extremity surgery within our institution, 2) to develop prescription guidelines, and 3 ) to educate patients about opioid usage and disposal.
Methods: Stage I consists of collecting prospective data from patients undergoing upper extremity surgery by 4 upper extremity surgeons over 6 consecutive months. Data are gathered from the electronic medical record and survey at clinic visits up to 90 days postoperatively including level of pain, opioid pills prescribed (converted to morphine equivalents), number of unused pills, and date of last opioid use. Patients are asked whether they were educated on pill disposal. Results will be used to develop prescription guidelines and patient education protocols.

Stage II consists of collecting prospective data from patients undergoing upper extremity surgery by 4 upper extremity surgeons over 6 consecutive months. Postoperative opioid prescriptions guided by previous results will be provided with standardized education on opioid disposal. The aforementioned data will again be collected at clinic visits up to 90 days postoperatively. Data from each stage will then be compared to assess the effectiveness of the implemented guidelines.

Results \& Conclusions: Pending further data collection.

https://doi.org/10.32873/unmc.dc.gmerj.1.1.037

\section{Identifying Current Opioid Prescription Practice and Implementing Guidelines after Total Knee and Total Hip Arthroplasty \\ Gordon Roedel, Phillip Thomas, Curtis Hartman, Kevin Garvin, Beau Konigsberg}

Mentor: Curtis Hartman

Program: Orthopaedic Surgery

Background \& Objectives: Opioid pain medications prescribed following total hip arthroplasty (THA) and total knee arthroplasty (TKA) are critical to postoperative pain management. However, a lack of evidence regarding appropriate prescription practices can contribute to overprescribing and diversion of opioids within the community. Objectives of this quality improvement project are 1) to understand prescribing habits and patient utilization of opioids following THA and TKA within our institution, 2) develop appropriate prescription guidelines, and 3) improve patient knowledge regarding unused opioid disposal.

Methods: Stage I consists of collecting prospective data from opioid-naïve patients undergoing primary THA and TKA over 6 consecutive months. Data are gathered from the electronic medical record and survey at clinic visits up to 90 days postoperatively including level of pain, number/type of opioid pills prescribed postoperatively (converted to morphine equivalents), number of unused pills, and date of last opioid use. Patients are asked whether they were educated on disposal of unused opioids. Results will be used to develop opioid prescription guidelines and patient education protocols which will be implemented during Stage II.

Stage II consists of collecting prospective data from opioid-naïve patients undergoing primary THA and TKA over 6 consecutive months. Postoperative opioid prescriptions guided by previous results will be provided with standardized education on opioid disposal. The aforementioned data will again 